\title{
The Interpretation of Shared Age-Related Variance among Factors in Cross-Sectional Cognitive Aging Studies
}

\author{
Kaarin Anstey \\ Australian National University, Canberra, Australia
}

\author{
Key Words \\ Aging $\cdot$ Cognition $\cdot$ Cross-sectional studies, \\ methodology
}

\begin{abstract}
Many cross-sectional correlational studies in cognitive aging have focused on explaining age-related variance. It has been assumed that variables sharing variance with both cognition and age may be the key explanatory variables underlying the cognitive decline in normal aging. Statistical biases intrinsic to this approach have been described by Hofer and Sliwinski and a narrow age cohort design proposed. The present paper aims at explaining how Hofer and Sliwinski's criticisms apply to a specific type of research design in cognitive aging where the goal is to identify underlying aging processes, but does not apply to more general gerontological research. Methods to estimate bias in cross-sectional studies are required as is greater awareness of this potential bias.
\end{abstract}

Copyright $@ 2002$ S. Karger AG, Base

In cognitive aging research, many studies have focused on identifying factors that share age-related variance in cognitive performance [e.g., 1-4]. The logic of this widely used research paradigm is that the variance in cognitive

\begin{tabular}{ll}
\hline KARGER & ( 2002 S. Karger AG, Basel \\
Fax +4161306 12 34 & \\
$\begin{array}{l}\text { E-Mail karger@karger.ch } \\
\text { www.karger.com }\end{array}$ & $\begin{array}{l}\text { Accessible online at: } \\
\text { www.karger.com/journals/ger }\end{array}$
\end{tabular}

performance due to age differences may be partitioned from variance due to individual differences that are unrelated to age. Age-related variance is assumed to represent variability that occurs as a result of one or more underlying aging processes. Progress in this field has been based in part on the idea that if we can identify variables that explain age-related variance, then we may be able to say something about the process or processes that explain cognitive aging. With respect to memory aging, Luszcz and Bryan [5] cite processing speed, executive function, and sensory function as possible mediators that have been examined. This logic of identifying mediators in terms of their capacity to explain age-related variance is applied within cross-sectional correlational studies. However, the data are unable to yield information on whether the variables that explain age-related variance are causally related to cognitive aging. However, inferences and theories may be developed on the basis of consistent results that then require testing using alternative methodologies such as experiments or longitudinal research. Examples of variables shown to explain large proportions of age-related variance in fluid intelligence include vision, hearing, muscle strength, processing speed, and working memory $[1,4$, 6]. In contrast, variables such as education consistently explain individual differences in cognitive performance in old age, but not age differences.

Hofer and Sliwinski [7] eloquently alert the reader to the many limitations of cross-sectional research and the

Kaarin Anstey

Centre for Mental Health Research

Australian National University

Canberra, ACT 0200 (Australia)

Tel. +612612 58410, Fax +612612 50733, E-Mail kaarin.anstey@anu.edu.au 
difficulty in making theoretical inferences based on mediational models of age-cognition relations. Hofer and Sliwinski [7] show that significant covariances between two unrelated variables in samples with a wide age range can occur, simply because of the variables joint association with age. They demonstrate how results are positively biased towards finding significant associations among variables when there is variation in age within the sample and the key variables of interest are associated with age. This means that the shared age-related variance among factors associated with cognitive performance may be in part due to a statistical artifact in cross-sectional studies. Thus, inferences about causality or even explanatory roles of variables statistically identified as mediators must be made with extreme caution and require validation with experimental and longitudinal studies. This provides another reason why causal inferences cannot be made confidently on the basis of cross-sectional mediational models.

It is important to place this valid criticism of crosssectional research in perspective. Firstly, this problem does not mean that all results showing shared age-related variance among variables associated with chronological age are wrong, but rather that the size of the associations may be inflated. The difficulty for researchers in this field at present is that we do not have a method for estimating the extent to which our results are biased. A useful interim approach to the problem of age aggregation is to report age-partialled results routinely to establish whether there is any association among variables that may be independent of age. Another factor to consider in relation to this criticism of cross-sectional research is that it is most problematic for studies attempting to identify underlying aging processes and least problematic for descriptive studies. Many valuable findings have resulted from cross-sectional cognitive aging research to date, such as the now classic distinction between fluid and crystallized intelligence [8]. Much research in gerontology is descriptive with respect to age. For example, prevalence studies aim to find out rates of disease at various ages. Other research in gerontology is not specifically aimed at explaining age differences or age changes, but instead seeks to understand phenomena that occur in later life, and hence the problem of partitioning variance on the basis of age does not occur.

Hofer and Sliwinski [7] propose the narrow age cohort design as an alternative method for cross-sectional designs employing a wide age range. Studying samples of individuals of the same age 'detrends' the population level effects of age differences. However, this method also has limitations. If there is no variation in age within a study, then it is not possible to study age or aging at all. Longitudinal follow-up of a narrow age cohort would overcome this problem, but longitudinal studies are themselves plagued by a range of methodological problems, with selective attrition being the most intransigent. The narrow age cohort design is useful where the experimenter aims at eliminating individual differences due to age within a sample. It may be less appropriate for answering many of the common types of descriptive questions that gerontologists ask. For example, much gerontological research answers questions about the nature of development of affect, personality or well-being at different ages, prevalence rates of activity, disease and disability, and the impact of life events.

The problems of cross-sectional research raised by Hofer and Sliwinski [7] are most pertinent when causal inferences are being made about factors explaining an aging process or mechanism. The narrow age cohort design is most useful in this context for testing hypotheses derived from traditional cross-sectional studies that have a wide age range. But even in this case, mechanisms and processes are dynamic entities that require longitudinal evaluation. Hofer and Sliwinski [7] rightly argue that multiple approaches and methodologies need to be applied to the study of a single problem in gerontology to attenuate misinterpretations stemming from the biases inherent in each method.

The problem of aggregation described by Hofer and Sliwinski is also relevant to theories of cognitive aging based on factor analyses that yield a common factor. Many studies have now demonstrated that variables correlated with chronological age will form a common factor in factor analytic models $[4,6,9]$. The common-factor solution is especially appealing, because it is consistent with the general principle of seeking parsimony in accounting for research findings in the behavioural sciences. However, Hofer and Sliwinski [7] do not distinguish between studies where variables that correlate with age are used to explain hypothesized aging processes and studies where variables that correlate with age are used as indices of biological or functional age.

The fact that cognitive and sensorimotor variables are known to be correlated with age has been exploited in functional age studies and in studies of biomarkers and cognition [10]. In these cases, variables are used as indices of age and not necessarily imbued with explanatory value. For example, Anstey and Smith [9] showed that a common factor of sensorimotor variables could replace age in a model of contextual factors associated with cognitive 
performance in a cross-sectional study. It is, therefore, important to appreciate that the age aggregation problem is critical when variables that statistically correlate with age are given theoretical importance as potential explanatory variables rather than as indices of age itself. In these situations experimental investigations are required to further test and evaluate hypotheses deriving from correlational studies, as has been done recently by Lindenberger et al. [11] with respect to the association between sensory and cognitive performance in old age or by Bryan et al. [12] with respect to the frontal hypothesis of cognitive aging. Perhaps cross-sectional correlational research examining factors that explain age-related variance should be viewed as a useful field for developing hypotheses, within a larger programme of research that employs a range of methodologies.

\section{References}

1 Anstey KJ, Lord SR, Williams P: Strength in the lower limbs, visual contrast sensitivity and simple reaction time predict cognition in older women. Psychol Aging 1997;12:137-144.

2 Bryan J, Luszcz MA: Speed of information processing as a mediator between age and freerecall performance. Psychol Aging 1996;11:39.

3 Salthouse TA, Hambrick DZ, McGuthry KE: Shared age-related influences on cognitive and non-cognitive variables. Psychol Aging 1998; 13:486-500.

4 Lindenberger U, Mayr U, Kliegl R: Speed and intelligence in old age. Psychol Aging 1993;8: 207-220.
5 Luszcz MA, Bryan J: Toward understanding age-related memory loss in late adulthood. Gerontology 1999;45:2-9.

6 Lindenberger U, Baltes P: Sensory functioning and intelligence in old age: A strong connection. Psychol Aging 1994;9:339-355.

7 Hofer SM, Sliwinski MJ: Understanding ageing: An evaluation of research designs for assessing the interdependence of ageing-related changes. Gerontology 2001;47:341-352.

8 Horn JL, Cattell RB: Age differences in fluid and crystallized intelligence. Acta Psychol (Amst) 1976;26:107-129.

9 Anstey KJ, Smith GA: Interrelationships among biological markers of aging, health, activity, acculturation, and cognitive performance in late adulthood. Psychol Aging 1999; 14:605-618.
10 Anstey KJ, Lord SR, Smith GA: Measuring human functional age: A review of empirical findings. Exp Aging Res 1996;22:245-266.

11 Lindenberger U, Sherer H, Baltes PB: The strong connection between sensory and cognitive performance in old age: Not due to sensory acuity reductions operating during cognitive assessment. Psychol Aging 2001;16:196-205.

12 Bryan J, Luszcz MA, Pointer S: The contribution of executive function and processing resources to age differences in the effects of strategy use on free recall. Aging Neuropsychol Cogn 1999;6:273-287. 